Revue des patrimoines

\title{
Du moteur à l'usine, ou petite histoire d'une grande entreprise nancéenne : la Compagnie Générale Electrique
}

\section{Pascal Thiébaut}

\section{OpenEdition}

Journals

Édition électronique

URL : http://journals.openedition.org/insitu/2993

DOI : 10.4000/insitu.2993

ISSN : 1630-7305

Éditeur

Ministère de la culture

Référence électronique

Pascal Thiébaut, « Du moteur à l'usine, ou petite histoire d'une grande entreprise nancéenne : la Compagnie Générale Electrique », In Situ [En ligne], 8| 2007, mis en ligne le 01 mars 2007, consulté le 19 avril 2019. URL : http://journals.openedition.org/insitu/2993 ; DOI : 10.4000/insitu.2993

Ce document a été généré automatiquement le 19 avril 2019

\section{(c)}

In Situ Revues des patrimoines est mis à disposition selon les termes de la licence Creative Commons Attribution - Pas d'Utilisation Commerciale - Pas de Modification 4.0 International. 


\title{
Du moteur à l'usine, ou petite histoire d'une grande entreprise nancéenne : la Compagnie Générale Electrique
}

\author{
Pascal Thiébaut
}

La création de la Compagnie Générale Electrique intervient dans un contexte local fortement marqué par le développement d'une industrialisation périurbaine dont l'empreinte dans le paysage est puissamment emblématique. Elle marque la convergence entre des intérêts régionaux et parisiens en vue de l'exploitation d'une technologie innovante, qui dépasse très rapidement le cadre local. Elle traduit enfin l'une des premières applications, en Lorraine non annexée, d'un plan-masse d'usine conditionné par l'emploi de l'électricité comme force motrice secondaire, auquel se surimposent des techniques constructives nouvelles.

\section{Le contexte industriel nancéen : atouts capitalistiques et forte implication des entrepreneurs}

2 L'industrialisation "lourde " de la ville intervient seulement à partir de 1872, au moment de l'annexion née du traité de Francfort d'une partie de la Lorraine par l'Empire allemand. Un vaste mouvement d'installation d'entreprises alsaciennes en Lorraine, restée française, s'amorce et touche principalement Nancy et les villes moyennes que sont Epinal et Saint-Dié (Vosges), Lunéville et Pont-à-Mousson (Meurthe-et-Moselle). Le prétexte patriotique mis en avant masque très partiellement la nécessité de conserver le marché français en s'affranchissant des droits de douane frappant les produits étrangers à leur entrée en France, malgré le tarif alors préférentiel accordé à l'Allemagne ${ }^{1}$. Cet afflux d'industriels concerne de nombreuses activités consommatrices d'énergie: transformation des métaux, tonnellerie, industrie textile, matériel d'équipement industriel, agro-alimentaire. Il contribue fortement au développement de l'industrie 
textile des Vosges, dont les prémices se font sentir en 1864-1865 au moment de l'ouverture des premières lignes de chemin de fer qui favorisent l'importation, en grandes quantités, du coton américain. La modernisation de ces entreprises, associée à des créations nouvelles qui interviennent dans les années 1890-1900, constituent un marché potentiel pour les machines d'équipement industriel et de force motrice.

La ville de Nancy est l'une des premières en France, avec Grenoble et Rouen, à bénéficier d'une distribution d'énergie électrique. C'est dans le courant de l'année 1887 qu'entre en service la première centrale électrique sous l'impulsion de l'architecte nancéen Félicien César (1849-1930), auteur de nombreuses usines ${ }^{2}$ dans la région ${ }^{3}$ et de l'ingénieur électricien Fabius Henrion (1859-?), constructeur de machines électriques. Implantée en centre-ville, la Station centrale d'électricité de Nancy produit, grâce à une dizaine de dynamos, du courant continu sous une tension de 100 volts. Il est pour l'essentiel consommé comme force motrice par des industriels ${ }^{4}$, une faible partie contribuant à l'éclairage public de quelques rues. Les actionnaires de la compagnie d'électricité se limitent à des entrepreneurs locaux, dont les établissements sont implantés dans le secteur d'industrialisation récente de la ville, soit à environ trois kilomètres vers l'ouest. La zone de lotissement industriel, orientée grossièrement nord-sud, s'étire sur une longueur de deux kilomètres, sur environ 500 mètres de largeur. Elle est desservie, depuis 1845, par le canal de la Marne au Rhin et par une voie ferrée de contournement ouest de la ville, ouverte en 1872. Son établissement se fait à l'instigation conjointe des industriels et du ministère de la Guerre qui lui assigne une vocation stratégique, à vingt-cinq kilomètres en ligne droite de la frontière allemande. Cette bande relativement étroite accueille rapidement de nombreuses firmes dont les plus importantes sont la verrerie Daum (1874) et la tonnellerie Fruhinsolz (1882). Elle abrite, en outre, un grand nombre de magasins et entrepôts à usage commercial qui bénéficient de raccordements aisés à la voie ferrée dans un secteur alors vierge de toute urbanisation ${ }^{5}$.

\section{La Compagnie Générale Electrique : une nouvelle société à vocations multiples}

4 La liquidation de la Station centrale d'électricité de Nancy, alors sous la raison sociale de Fabius Henrion et Cie intervient dans les premiers mois de l'année 1898. L'entreprise présente un certain nombre de handicaps, liés notamment à sa situation dans un espace urbanisé dense qui lui interdit toute extension future et à son éloignement des principaux centres de consommation d'énergie électrique, ce qui la contraint à l'entretien d'un réseau de distribution souterrain de près de neuf kilomètres. Le transport de courant continu impose de surcroît de nombreuses contraintes d'isolement difficilement compatibles avec des agrandissements potentiels, au moment où l'emploi des courants alternatifs est mieux maitrisé. Cette nouvelle technologie se répand de plus en plus dans l'industrie, notamment en Suisse et en Allemagne, pays exportateurs de matériel électrique en France, dès le début des années 1890. La commodité d'emploi de ces nouvelles machines trouve rapidement la faveur des industriels de l'Est de la France.

Les actifs de la société Fabius Henrion et Cie sont adjugés le 12 juillet 1898, à la Compagnie Générale d'Electricité. Créée à Paris ${ }^{6}$ le 31 mai 1898, cette dernière fournit en distribution électrique les villes moyennes françaises, en s'inspirant de l'Allgemeine Elektrizität Gesellschaft (A.E.G.), établie en Allemagne en 1884. Son fondateur, le centralien Pierre 
Azaria (1865-1953), qui obtient au sortir de l'Ecole le poste alors jugé peu enviable de directeur de l'usine d'électricité de Rouen, développe et modernise rapidement cette affaire. Dès 1899, il met en chantier la nouvelle usine de Nancy, en bordure immédiate ouest du canal. Confiés à l'architecte Félicien César, les travaux de construction sont rapidement menés ; la disposition d'ensemble facilite l'approvisionnement de l'usine en charbon pour les turbines à vapeur. A la même période débute la réalisation du tramway de Nancy sous la direction de l'architecte-ingénieur polytechnicien Henry Gutton (1874- ? ), neveu de l'architecte Henri Gutton (1851-1933) dont il sera question plus loin.

6 Par ailleurs, l'idée de créer une usine de construction de matériel électrique à Nancy est dans l'air dès 1889, peut-être à l'instigation de Fabius Henrion; la question agite régulièrement les milieux intellectuels et artistiques proches de l'Ecole de Nancy. Dans les premiers mois de l'année 1898, une souscription de 2000 actions d'un montant de 1000 francs chacune est lancée et rencontre un succès considérable; le 30 mars la décision de fondation est prise définitivement. Le 4 juin 1898 naît la Compagnie Générale Electrique ${ }^{7}$, société ayant pour objet principal la production de moteurs et d'équipements électriques divers pour l'industrie. La coïncidence de dates et de dénomination n'est pas fortuite : ses instigateurs recherchent manifestement une complémentarité technologique avec la précédente et surtout une forme de concurrence dissimulée dans le domaine de la fabrication et du transport de l'électricité.

7 La première assemblée des actionnaires intervient le 22 juin, juste avant la vente de la société Fabius Henrion et Cie. Elle est composée principalement d'industriels : le filateur nancéen Alphonse Francin (1870-1914), Frédéric Dyckhoff, constructeur de moteurs ${ }^{8}$ à Bar-le-Duc (Meuse), Léopold Betting, brasseur à Maxéville près de Nancy, et le tonnelier Adolphe Fruhinsolz. Y figurent aussi des architectes locaux : Félicien César, Emile André (1871-1933), le célèbre architecte de l'Ecole de Nancy Charles Schuler (1852-1900), Henri Gutton, autre figure de l'Ecole de Nancy, et des entrepreneurs de travaux publics: Auguste Bichaton, Jean-Baptiste France-Lanord. La présidence de la société est dévolue à Alphonse Francin, la totalité du capital se monte à 2 millions de francs, dont 1850000 sont aux mains d'un des deux administrateurs délégués, Charles Vicarino (1862-1913). Natif de Mohilev (Russie), mais issu d'une famille d'ingénieurs d'origine suisse, et ancien élève de l'Ecole polytechnique de Zurich, Vicarino n'est pas un inconnu dans le domaine de l'électricité. Etabli à Nancy depuis plusieurs années, il fait figure de novateur dans le domaine des techniques électriques. On lui doit notamment les premiers essais, couronnés de succès, d'éclairage de voitures de chemin de fer à partir de génératrices en prise directe avec les arbres des roues (1897-1898). Il est aussi à l'origine de la création en 1904 de la société d'éclairage de la ville d'Esch-sur-Alzette (Luxembourg) et en 1913 de la société des Chocolats Stanislas, dont l'usine, située à proximité de Nancy ${ }^{9}$, est construite la même année.

\section{Une usine imposante et moderne}

8 “Des constructions imposantes ont été élevées à Nancy dans les vastes terrains qui bordent la rue Oberlin entre le canal de la Marne au Rhin et le chemin de fer de ceinture de Nancy Saint-Georges" lit-on en 1900 dans une revue d'art nancéenne ${ }^{10}$, support inhabituel pour relater ce type d'événement. Les collaborateurs de cette revue, qui traite largement des innovations stylistiques liées au développement de l'Art nouveau à Nancy, figurent parmi les actionnaires de l'affaire; ils soulignent aussi la perception nouvelle 
qu'introduit dans un paysage encore rural un édifice offrant une architecture alliant fonctionnalité, lisibilité et austérité, tempérée par des éléments de décor particulièrement soignés.

La direction de la construction est confiée à l'architecte Henri Gutton, auquel succède Henry Bertrand, auteur en 1913 des magasins de la Belle Jardinière à Nancy ${ }^{11}$. Les travaux de maçonnerie sont conduits par l'entreprise nancéenne France-Lanord et Bichaton, dont les dirigeants sont d'éminents actionnaires de l'entreprise. Menée avec rapidité, l'édification des principaux bâtiments débute fin juin 1898, pour s'achever le 21 janvier de l'année suivante. L'ampleur considérable du chantier nécessite, pour l'acheminement des matériaux de construction, l'établissement d'un port temporaire d'une longueur de quarante-quatre mètres sur le canal en face de l'usine.

L'édifice terminé occupe partiellement, pour se réserver des possibilités ultérieures d'extension, une emprise de terrain de 400 mètres de longueur grossièrement orientée ouest-est sur une largeur de 150 mètres. La parcelle de 47000 mètres carrés est délimitée, au sud, par la rue Oberlin qui suit le tracé en courbe du canal et, au nord, par la voie ferrée de contournement de Nancy (fig. $\left.\mathbf{n}^{\circ} \mathbf{1}\right)$.

Figure 1

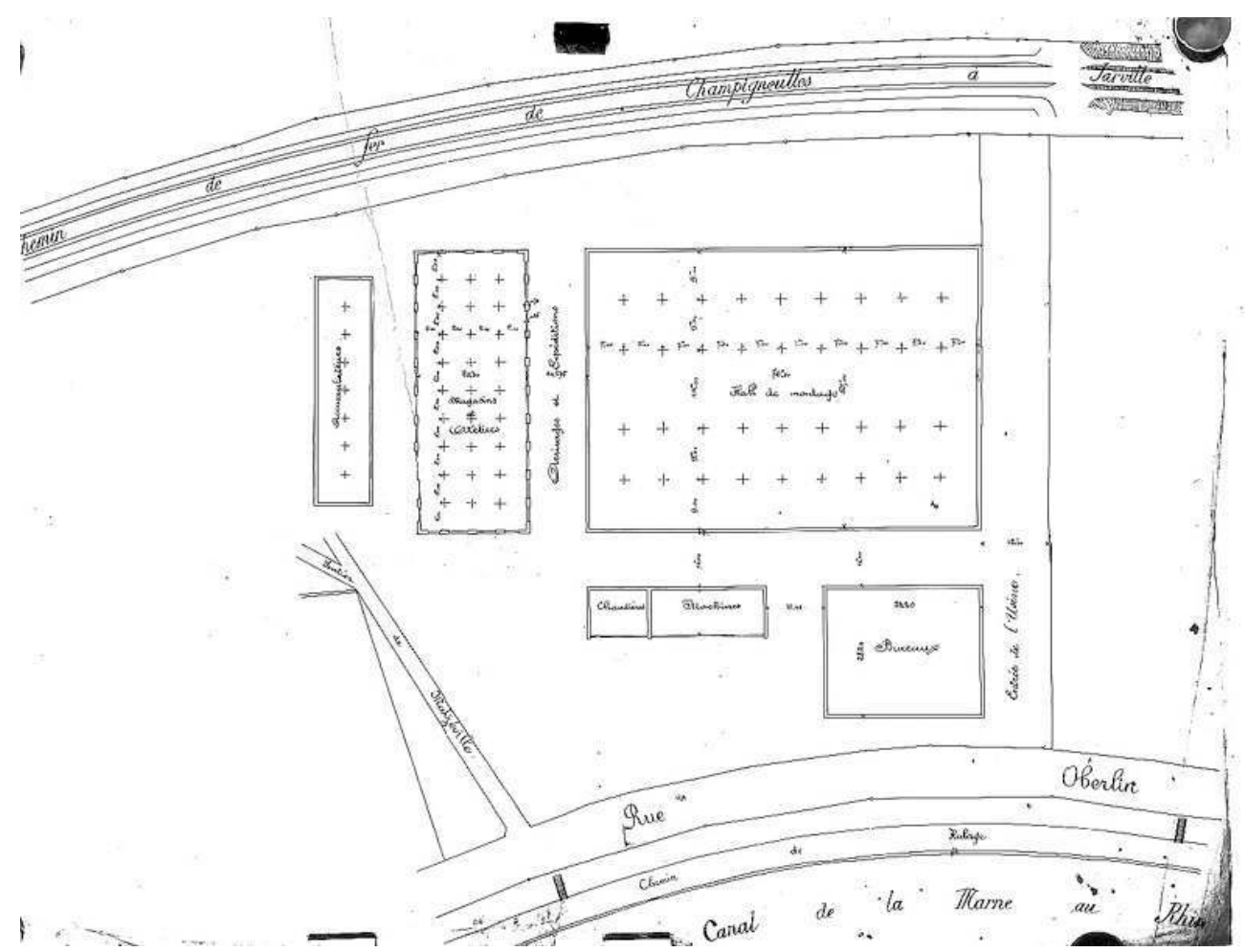

Plan, non daté [1898]. Lempereur, S. @ Inventaire général, ADAGP/Entreprise France-Lanord et Bichaton, 1998

Elle comprend alors un atelier de fabrication à trois vaisseaux, dont le plus haut, au centre, abrite le pont roulant; un magasin de pièces détachées dont le toit-terrasse est aménagé en réserve d'eau ; une chaufferie et une salle des machines à côté desquelles se trouve le bâtiment administratif (fig. $\mathbf{n}^{\circ} \mathbf{2}$ ). 


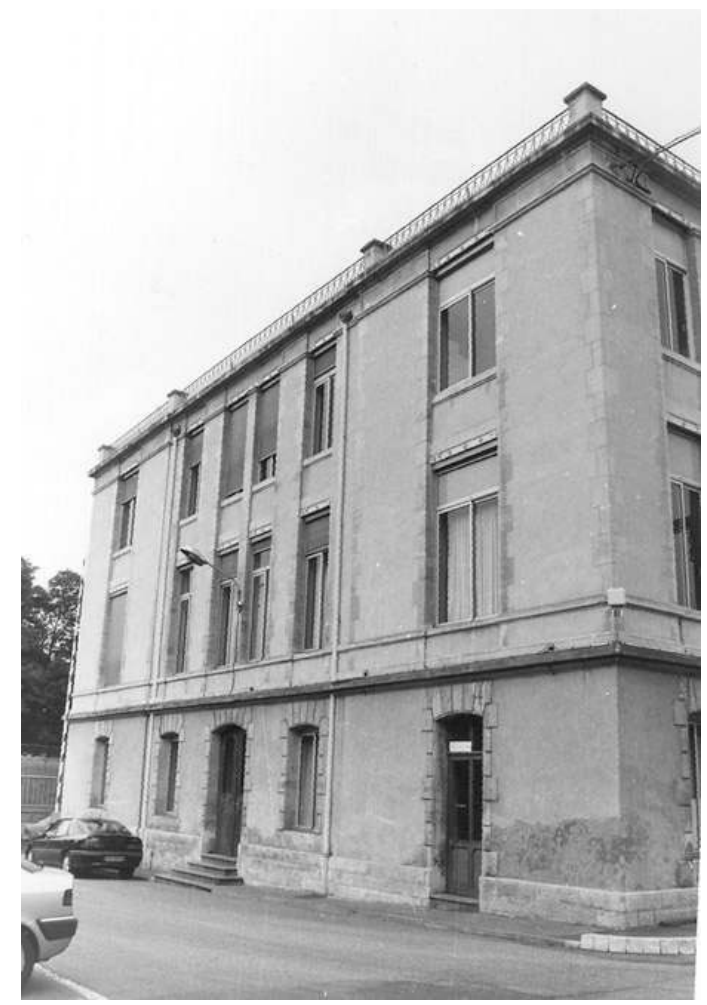

Bureau et bureau d'études, élévation Est. Thiébaut, Pascal @ Inventaire général, ADAGP, 1998

Ce dernier est pourvu de deux étages carrés, et surmonté d'un toit en terrasse avec lanterneau vitré central donnant jour à la cage d'escalier. Il offre au regard un volume cubique percé d'ouvertures à linteaux en fer IPN aux étages, tandis qu'au rez-dechaussée, les encadrements de baies présentent des linteaux en pierre de taille à pseudocrossettes $^{12}$. L'atelier de fabrication possède des ouvertures cintrées en briques rouges, à claveaux et écoinçons en pierre calcaire, selon un schéma encore traditionnel. 


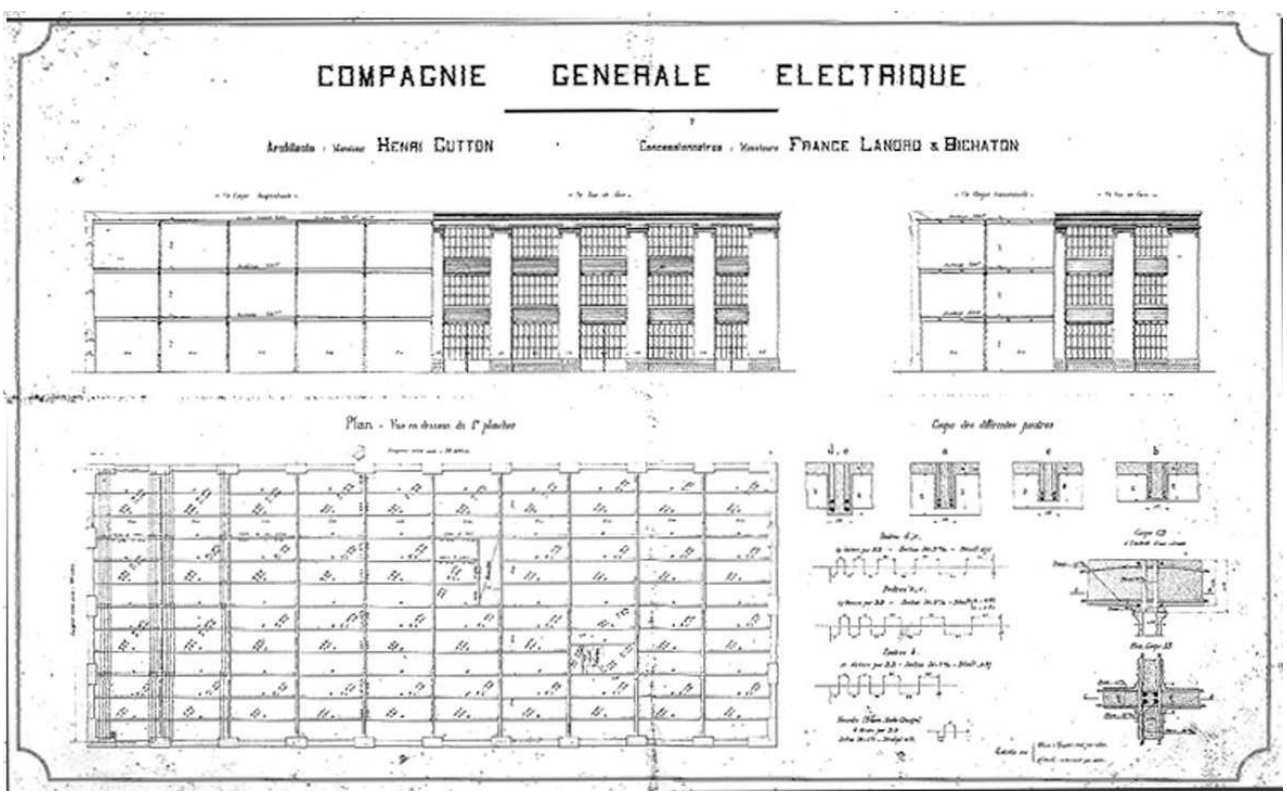

Magasin de pièces, plan, coupe et élévation, 1898. George, Alain

(c) Inventaire général, ADAGP, 1999/Entreprise France-Lanord et Bichaton.

13 Le magasin (aujourd'hui disparu) (fig. $\mathbf{n}^{\circ}$ 3) offrait une élévation sur trois niveaux, ponctuée à intervalles réguliers de piles massives en brique dégageant de grandes baies vitrées. L'originalité de l'édifice réside d'une part dans l'adoption d'un plan-masse où la chaufferie et la salle des machines sont dissociées de l'atelier de fabrication, disposition qu'autorise l'emploi de l'électricité, abandonnant ainsi la transmission de force motrice par arbres ou câble ${ }^{13}$, et d'autre part dans la mise en place de procédés de construction novateurs. Les séparations de niveaux sont réalisées en béton armé, selon le procédé Hennebique $^{14}$; on retrouve ce dispositif dans la terrasse du magasin dont les bords intérieurs sont munis d'un glacis pentu formant brise-lame, afin d'éviter l'ébranlement qui pourrait se produire sur la maçonnerie du bâtiment sous l'effet d'un vent violent.

Le hall de fabrication est pourvu d'une charpente métallique reposant sur des piliers à treillis rivetés, fournis par l'entreprise F. Schertzer ${ }^{15}$ de Nancy (fig. $\mathbf{n}^{\circ} \mathbf{4}$ ). 


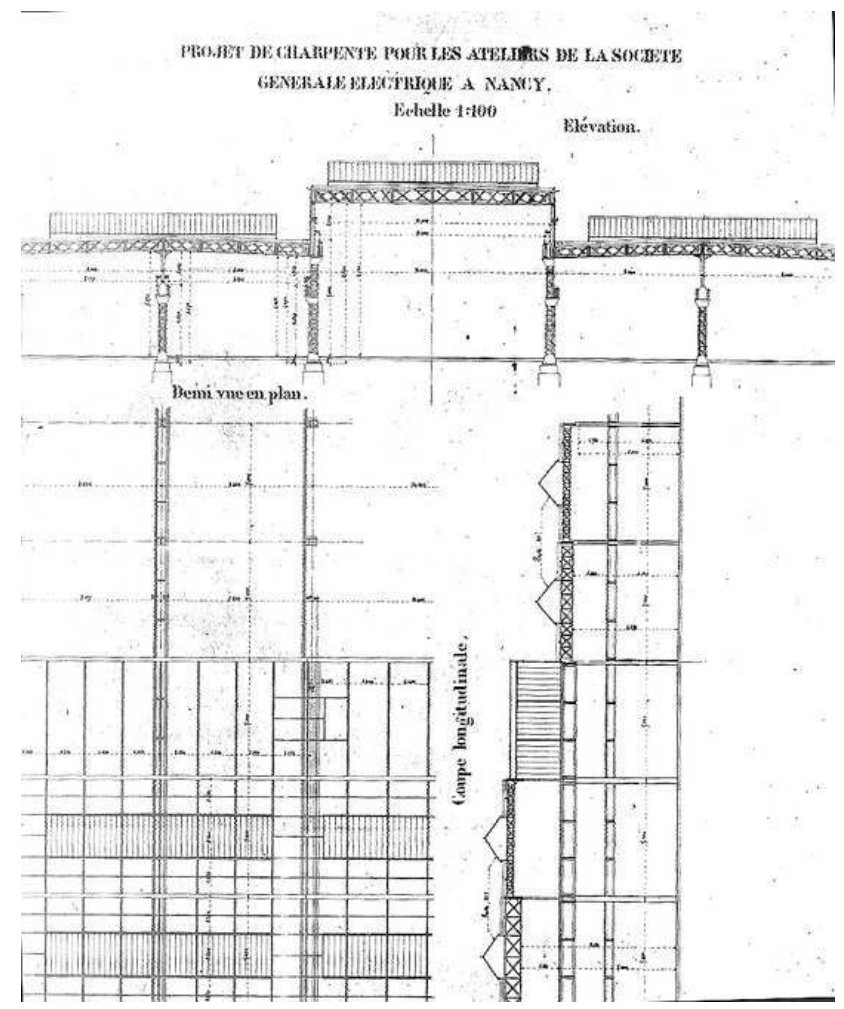

Atelier de fabrication, plans et coupes de la charpente en fer, 1/100e, par F. Schertzer, 1898

(C) Inventaire général, ADAGP, 1998/Entreprise France-Lanord et Bichaton.

C'est l'un des premiers emplois connu dans la région d'une structure d'une telle ampleur et d'une telle portée (50 mètres) ${ }^{1617}$. La toiture des vaisseaux latéraux comporte des lanterneaux vitrés reposant sur une couverture à faible pente, à l'origine en ciment volcanique ${ }^{18}$. Le vaisseau central, affecté au montage des dynamos et des moteurs, est équipé d'un pont-roulant de cinq tonnes, les vaisseaux latéraux abritent les machinesoutils et des ponts-roulants secondaires de plus faible dimension. Des moteurs électriques commandent, en bout de vaisseau, les arbres de transmission qui entraînent les machines-outils par l'intermédiaire de courroies débrayables et de roues à diamètre variable pour le réglage de la vitesse. L'heure est donnée par des pendules électriques commandées par un régulateur central.

Un second hall de fabrication est construit en 1906, à l'ouest du premier, mais selon une orientation différente. Cette réalisation présente une façade principale courbée pour suivre le tracé de la voirie sur la rue Oberlin (fig. $\mathbf{n}^{\circ} 5$ ). 
Figure 5

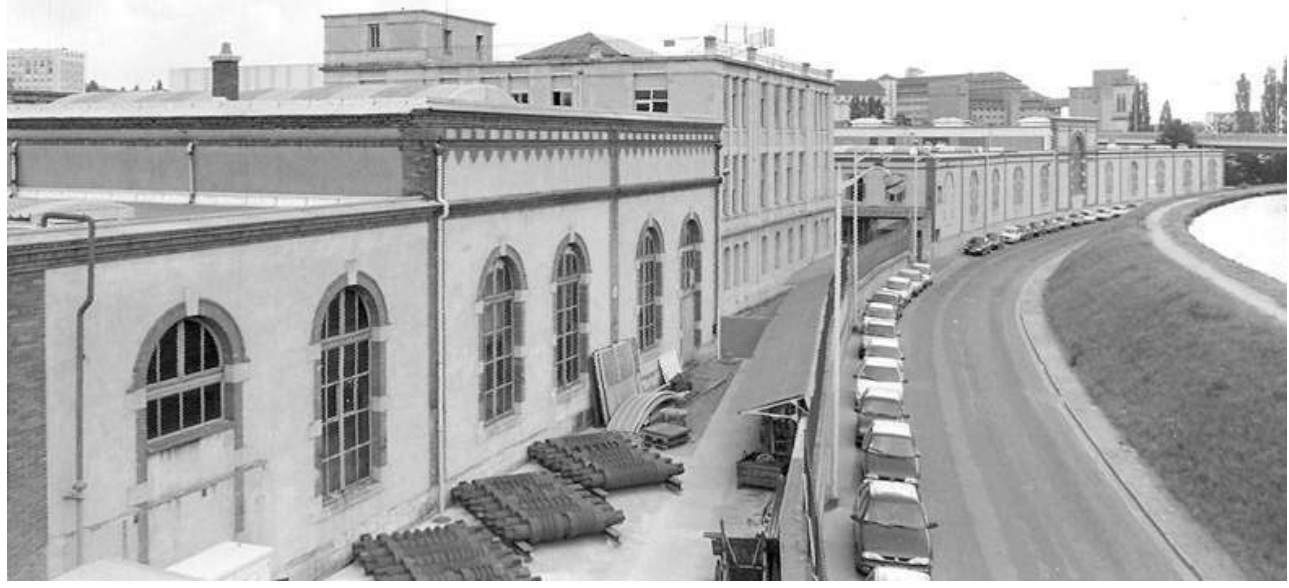

Ensemble de la façade sur la rue Oberlin, vue depuis l'ouest. Thiébaut, Pascal

(c) Inventaire général, ADAGP, 1998.

Ses trois vaisseaux sont traités, astuce autant technique qu'esthétique, à la manière d'une travée serlienne. Cette disposition particulière lui confère encore une élégance qui la signale fortement dans le paysage urbain environnant. Des extensions supplémentaires sont effectuées en 1911, 1918-1919 (sous la direction de l'architecte Henry Bertrand), 1920, 1929-1930, mais en respectant à chaque fois le parti initial, ce qui lui assure une unité de style peu courante dans un édifice à caractère industriel.

Le bâtiment à vocation administrative abrite une décoration intérieure d'une qualité exceptionnelle pour une entreprise industrielle, qui s'inscrit dans une mouvance tout à fait nouvelle, elle aussi. Un vaste hall d'entrée, pavé de carreaux de grès à motifs multicolores et pourvu de portes dont les vitres sont ornées de motifs floraux gravés à l'acide (fig. $\mathbf{n}^{\circ} \mathbf{6}$ ), donne accès à une vaste cage d'escalier éclairée par une verrière bombée sur plan carré (fig. $\mathbf{n}^{\circ} 7$ ). 


\section{Figure 6}

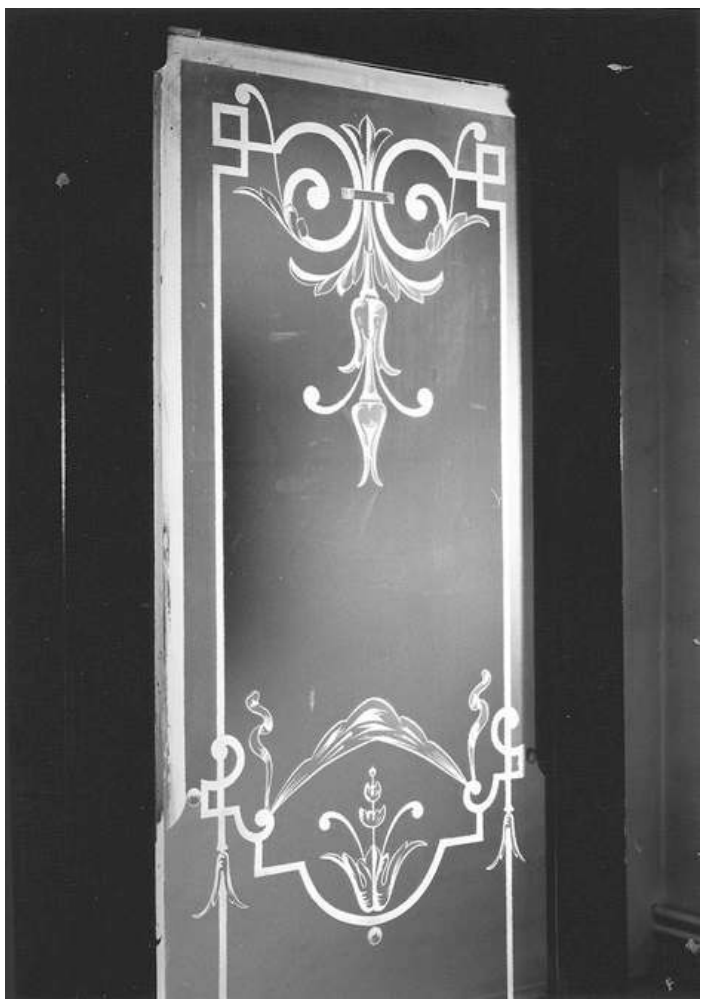

Bureau et bureau d'études, vue intérieure : porte vitrée en verre gravé. George, Alain (c) Inventaire général, ADAGP, 1998. 


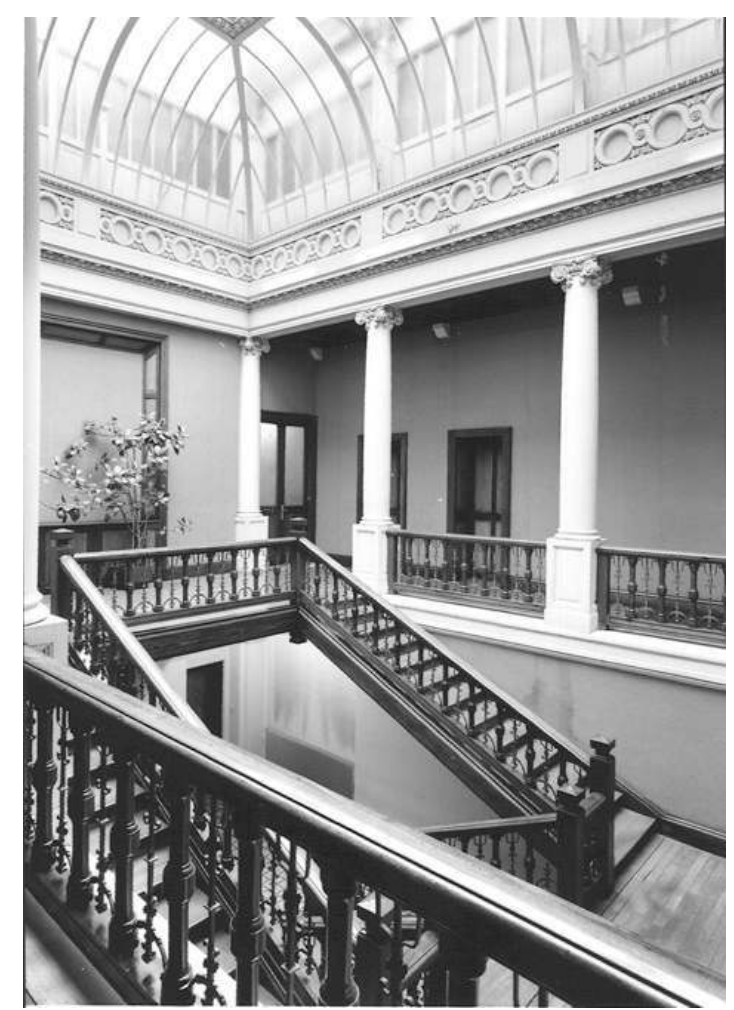

Bureau et bureau d'études, vue intérieure : cage d'escalier. George, Alain @ Inventaire général, ADAGP, 1998.

19 Elle dessert une partie des bureaux du premier étage, dont les portes vitrées ou à impostes vitrées s'ouvrent sur une coursive. La verrière, supportée par des colonnes de fonte ${ }^{19}$, masquées depuis 1918 par des colonnes renflées en tôle peinte pourvues de chapiteaux ioniques à petites guirlandes florales, présente un replat central délimité par une frise d'oves.

La menuiserie des balustrades de l'escalier et de la coursive est agrémentée de ferronneries. Cette distribution autour d'un puits de lumière parait s'inspirer des bureaux des usines Wendel de Hayange (1892), et de ceux du carreau de la mine de houille SaintCharles à Petite-Rosselle. Le programme de décor intérieur du bâtiment associe des ornements traditionnels, encore en vogue à la fin du XIXe siècle, et des éléments Art nouveau de l'Ecole de Nancy.

21 L'ébéniste Eugène Vallin (1856-1922) a réalisé les lambris (fig. $\mathbf{n}^{\circ}$ 8), portes, et étagères des deux bureaux réservés au directeur et à l'administrateur délégué ${ }^{20}$. Il est également l'auteur du mobilier d'une des pièces : table de travail, sièges et horloge de parquet ${ }^{21}$. Une pièce voisine abrite des menuiseries (porte, lambris à hauteur d'appui) de l'ébéniste Louis Majorelle (1859-1926). 


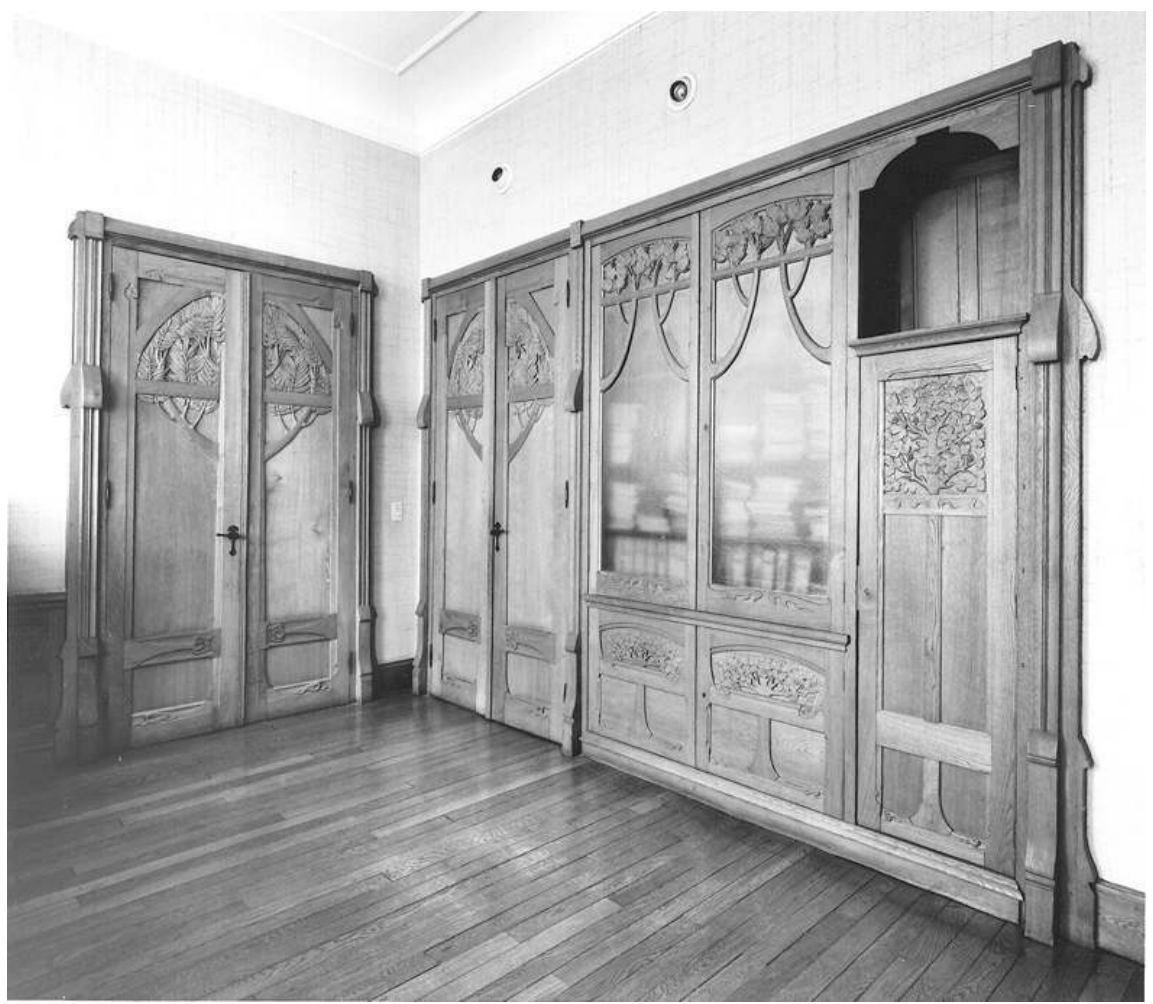

Bureau et bureau d'études, vue intérieure : bureau d'administrateur, portes et bibliothèque par E. Vallin. George, Alain

(c) Inventaire général, ADAGP, 1998

Dire que l'usine de la Compagnie Générale Electrique a fait école paraît hasardeux, mais on peut cependant affirmer que son édification recourt, pour la première fois, à des procédés de construction nouveaux, dont la combinaison dans un programme cohérent induit une morphologie encore très inhabituelle.

\section{Une entreprise innovante très impliquée dans l'industrialisation régionale}

Les premiers moteurs sont construits dans un atelier provisoire dépendant de l'usine textile d'Alphonse Francin ; la production dans l'usine terminée débute courant mai 1899. En 1900, la Compagnie Générale Electrique acquiert l'ancien moulin de Frouard, à dix kilomètres au nord de Nancy, pour y fabriquer des accumulateurs électriques de marque French-Willard, destinés notamment à équiper les tramways de Nancy ${ }^{22}$. Partiellement reconstruit en 1902, à la suite d'un incendie, cet ancien moulin fournit aussi par ligne aérienne la Compagnie Générale Electrique en énergie triphasée.

Dans le courant de l'année 1900, cette dernière produit le matériel de la centrale d'Eschsur-Alzette et une machine à courant continu de 500 ch destinée à la Compagnie Générale d'Electricité pour sa nouvelle centrale électrique; en 1902 elle obtient le marché d'équipement du tramway de Gérardmer-la-Schlucht (Vosges) dont les études ont été effectuées par Henry Gutton. En 1904, elle construit le premier alternateur triphasé de $1600 \mathrm{~kW}$, puis assure, entre 1905 et 1907, l'équipement électrique des aciéries et laminoirs 
de la société de Châtillon-Commentry à Neuves-Maisons, à dix kilomètres au sud-ouest de Nancy. Peu avant 1914, la concurrence commence à s'organiser avec notamment la création, à Nancy même, des Constructions Electriques de Nancy sous l'impulsion de l'ingénieur Maeder.

A partir de 1918, la société fournit de nombreuses entreprises textiles vosgiennes, dont la reconstruction commence, bénéficiant d'un mouvement amorcé à partir de 1902, lors d'importantes campagnes de modernisation de bâtiments industriels. En 1921, la Compagnie Générale Electrique lance un nouveau produit: le moteur asynchrone synchronisé. Son inventeur ${ }^{23}$, Jean Le Monnier, ami d'Emile Gallé (1846-1904), n'est autre que le fils de l'un des actionnaires fondateurs de l'entreprise.

La firme est rachetée en 1957 par la société suisse Les Ateliers de Sécheron, après un dépôt de bilan intervenu l'année précédente. En 1968, elle est rachetée par la Compagnie Electro-Mécanique (C.E.M.), puis par Alsthom ${ }^{24}$ en 1983. Depuis cette date, l'établissement de la rue Oberlin s'est progressivement spécialisé dans la réalisation de moteurs de bateau et notamment de " pod " (moteurs extérieurs aux coques) destinés aux navires de croisières. Face à de nombreuses contraintes d'espace et surtout d'accès, dues à sa présence dans un milieu urbain dense, l'entreprise a abandonné ses locaux d'origine courant 2001-2002, pour installer une nouvelle usine à Frouard, au bord de la Moselle canalisée, pas très loin du moulin qu'elle avait acquis en 1900.

Placée à un point de convergence dans les domaines techniques et industriels, la Compagnie Générale Electrique met en avant, dès sa constitution, l'importance du rôle joué par des capitaux dans une ville moyenne: ses actionnaires principaux en sont les constructeurs et les futurs consommateurs de produits finis ; l'actionnaire majoritaire est l'ingénieur-inventeur. De fortes personnalités révèlent cette complexité : l'architecteingénieur Henri Gutton et son neveu, concepteurs de réseaux de tramways, les entrepreneurs France-Lanord et Bichaton, eux-mêmes actionnaires de nombreuses entreprises qui se fournissent en moteurs sortant des ateliers de la Compagnie Générale Electrique et constructeurs d'usines selon les mêmes procédés de construction entraînant des formes architecturales identiques (fig. $\mathbf{n}^{\circ} \mathbf{9}$ ). 


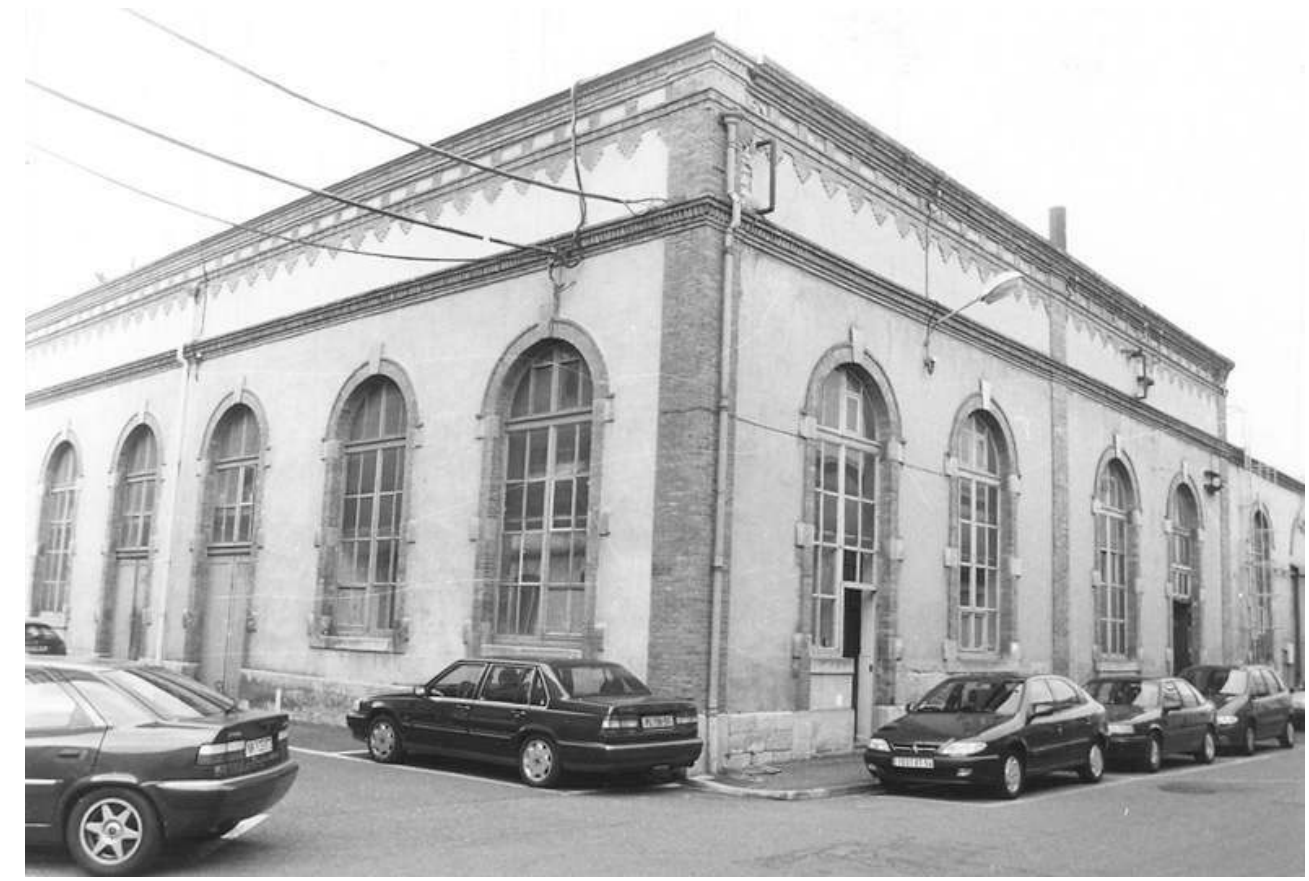

Elévation sur cour de la chaufferie et de la salle des machines : ouvertures et jeux de matériaux. Thiébaut, Pascal

(C) Inventaire général, ADAGP, 1998

C'est l'exemple même de l'entreprise d'essence locale qui va essaimer au plan national, à travers les nombreuses succursales qu'elle ouvre en France à partir des années 1920. L'édification de ses locaux semble marquer un tournant chronologique perceptible dans la région: le début d'une forme de production en série, donc peut-être d'appauvrissement, de l'architecture industrielle, à travers la généralisation progressive de procédés de construction identiques entraînant des morphologies proches. Il convient cependant d'en nuancer la portée; l'emploi de procédés de construction nouveaux se traduit par la réalisation d'édifices où la primauté de l'adaptation fonctionnelle n'entraine pas l'abandon d'une recherche esthétique qui s'illustre davantage par l'usage différencié des matériaux, de facture industrielle donc moins coûteuse, que par l'emploi d'un décor sophistiqué et ostentatoire. La baie cintrée laisse progressivement la place au linteau en fer apparent. L'usine est représentative de schémas morphologiques "lourds" qui vont s'accentuer dans les années suivantes: volumes cubiques des bâtiments accentués par la faible émergence des toits en terrasse ou à très faible pente; emploi de grandes surfaces vitrées en élévation qu'autorise l'emploi du béton armé; éclairage zénithal des ateliers par lanterneaux vitrés multiples. Le reflux du décor Art nouveau à l'intérieur du bâtiment administratif va tout à fait à l'encontre d'une pratique courante de l'architecture nancéenne de cette période qui en affiche les éléments sur les façades, au détriment d'intérieurs, traités bien souvent dans un goût plus conventionnel. Cette correspondance avec une technologie innovante n'est-elle pas plus subtile qu'il n'y parait? ? De nombreux objets Art nouveau ne doivent-ils pas leur existence à l'emploi de l'électricité? 


\section{NOTES}

1. Ce n'est plus le cas après 1892.

2. Voir dans la base Mérimée : notices IA54000926, IA54000947, IA54000016.

3. De Maxéville et Champigneulles. F. César se qualifie “d'architecte industriel ".

4. Jusqu'en 1906, le monopole de l'éclairage est accordé aux usines à gaz.

5. Ouverte en 1852, la voie ferrée Paris-Strasbourg, passant à trois kilomètres à l'ouest, n'a pratiquement pas suscité d'implantations industrielles.

6. Le siège social est situé 5, rue Boudreau à Paris. Il déménage en 1914 au 54, rue de la Boëtie.

7. Fonds de l'entreprise C.G.E. Alsthom, Nancy.

8. On lui doit la réalisation du premier moteur Diesel construit en France (C.N.A.M.).

9. Commune d'Essey-lès-Nancy; désaffectés, les locaux de belle facture sont occupés par le C.R.E.P.S.

10. La Lorraine Artiste, 1er août 1900, p. 19.

11. Peut-être le frère d'Ernest-François, né en 1876 à Paris, auteur de l'agrandissement du magasin de la Belle Jardinière de Paris.

12. La maison d'Henri Gutton, construite pour lui-même, présente ce type de décor.

13. Disposition encore fréquente dans les usines textiles construites après 1900.

14. Les premiers brevets sont pris en 1890 et 1892 . C'est en 1896 qu'est construit à Nancy le premier plancher établi selon ce principe.

15. L'ingénieur nancéen Frédéric Schertzer a travaillé à la grande galerie-promenoir de l'établissement thermal de Vittel (Vosges) [1897-1905], qui reliait les deux pavillons abritant les sources. Voir l'article de Bernard Toulier dans la revue In Situ, $n^{\circ} 7$, rubrique Varia : “ Un parfum d'Orient au cœur des villes d'eau" :

16. Les Ets. Daydé et Pillé de Creil (Oise) construisent en 1898 aux cristalleries de Baccarat une des premières grandes halles d'une portée supérieure à trente mètres dans la région.

17. Les Ets. Daydé et Pillé de Creil (Oise) construisent en 1898 aux cristalleries de Baccarat une des premières grandes halles d'une portée supérieure à trente mètres dans la région.

18. Louis Pack, entrepreneur à Nancy, concessionnaire d'un brevet déjà ancien déposé en 1832 (brevet Haeusler).

19. Fonds C.G.E. Alsthom, Nancy. Etat original.

20. Cette disposition n'était pas prévue sur les plans originaux du bâtiment, certainement réalisés en 1898. La pose des boiseries nécessite le déplacement d'une cheminée.

21. Fabriquée et livrée en 1903. Le mouvement à remontage à poids (avec train de sonnerie), provient des Ets. Manufrance de Saint-Etienne (Loire).

22. Afin de permettre le franchissement de faibles portions du tracé non pourvues d'alimentation aérienne par fil.

23. Brevet $n^{\circ} 495670$ délivré le 7 juillet 1919.

24. Créée en novembre 1928 par mise en commun des moyens de production de la division électrique de la Société Alsacienne de Constructions Mécaniques (S.A.C.M.) de Belfort (90) et des établissements Thomson-Houston-France. 


\section{RÉSUMÉS}

La révolution industrielle de l'électricité se met en place dans les deux dernières décennies du XIXe siècle. Ville moyenne, Nancy n'échappe pas à ce mouvement qui gagne l'Europe entière et les Etats-Unis et qui nécessite des capitaux importants. Puissant facteur d'auto-industrialisation, l'usage de l'électricité comme force motrice dans les usines induira de nouvelles habitudes de productions industrielles et une nouvelle division du travail. C'est dans ce contexte qu'est fondée en 1898 à Nancy la Compagnie Générale Electrique dont l'objet principal est la construction de moteurs. La conception très moderne de son usine symbolise ces nouvelles tendances.

The industrial revolution of electrification was played out during the last two decades of the 19th century. Nancy, a medium-sized city, was affected by this revolution which spread across the whole of Europe and the United States, and which was characterised by the importance of the capital investment it required. Electricity used as motive power for production was a powerful incentive to industrial development and the arrival of electric motors in factories brought about new styles of production and a new division of labour. This is the context in which the Compagnie Générale Electrique was founded at Nancy in 1898, a firm primarily devoted to the construction of electric motors. The modern design of its factory was itself emblematic of the new industrial trends.

\section{INDEX}

Mots-clés : art nouveau, béton armé Hennebique, Charles Vicarino, Ecole de Nancy, Georges Vallin, Louis Majorelle, mobilier, moteur asynchrone, moteur synchronisé, usine de matériel électrique

Keywords : Compagnie Générale Electrique, electric motors, electrification, Nancy

\section{AUTEUR}

\section{PASCAL THIÉBAUT}

Chercheur. Chargé du patrimoine industriel auprès du service chargé de l'inventaire général du patrimoine culturel, Région Lorraine. pascal.thiebaut@cr-lorraine.fr 